\title{
Surface-bound selectin-ligand binding is regulated by carrier diffusion
}

\author{
Ganyun Sun $\cdot$ Yan Zhang $\cdot$ Bo Huo $\cdot$ \\ Mian Long
}

Received: 23 October 2008/Revised: 6 February 2009/Accepted: 15 February 2009/Published online: 10 March 2009

(C) European Biophysical Societies' Association 2009

\begin{abstract}
Two-dimensional (2D) kinetics of receptorligand interactions governs cell adhesion in many biological processes. While the dissociation kinetics of receptorligand bond is extensively investigated, the association kinetics has much less been quantified. Recently receptorligand interactions between two surfaces were investigated using a thermal fluctuation assay upon biomembrane force probe technique (Chen et al. in Biophys J 94:694-701, 2008). The regulating factors on association kinetics, however, are not well characterized. Here we developed an alternative thermal fluctuation assay using optical trap technique, which enables to visualize consecutive bindingunbinding transition and to quantify the impact of microbead diffusion on receptor-ligand binding. Three selectin constructs (sLs, sPs, and PLE) and their ligand P-selectin glycoprotein ligand 1 were used to conduct the measurements. It was indicated that bond formation was reduced by enhancing the diffusivity of selectin-coupled carrier, suggesting that carrier diffusion is crucial to determine receptor-ligand binding. It was also found that $2 \mathrm{D}$ forward rate predicted upon first-order kinetics was in the order of sPs $>$ sLs $>$ PLE and bond formation was history-dependent. These results further the understandings in regulating association kinetics of surface-bound receptor-ligand interactions.
\end{abstract}

Keywords Association kinetics - Regulating factors · Carrier diffusion · Optical trap · Selectin · Ligand

G. Sun $\cdot$ Y. Zhang $\cdot$ B. Huo $\cdot$ M. Long $(\bowtie)$

National Microgravity Laboratory and Center for Biomechanics and Bioengineering, Institute of Mechanics, Chinese Academy of Sciences, 100190 Beijing, People's Republic of China e-mail: mlong@imech.ac.cn

\section{Introduction}

Cell adhesion mediated by receptor-ligand interactions is crucial to such biological processes as inflammatory reaction (Springer 1995), tumor metastasis (Albelda 1993), arteriosclerosis (Galkina and Ley 2007), and wound healing (Grinnell 1992). To mediate cell adhesions, receptors and their ligands must be anchored onto two apposed surfaces, which is so-called two-dimensional (2D) interaction. This is different from three-dimensional (3D) interaction where at least one of receptors and ligands is in fluid phase (Bell 1978). 2D association and dissociation kinetics governs the formation and rupture of surfacebound receptor-ligand bond. Direct measurement of 2D kinetics rates and governing factors is indispensable to understand the biophysical bases of receptor-ligand interactions in regulating cell adhesions.

2D dissociation kinetics (Hammer and Lauffenburger 1987; Alon et al. 1995; Chen et al. 1997) and forced bond rupture (Florin et al. 1994; Dammer et al. 1996; Tees et al. 2001) as well as their regulating factors (Evans et al. 2001; Levin et al. 2001; Huang et al. 2004; Marshall et al. 2005; $\mathrm{Wu}$ et al. 2007) have been investigated theoretically and experimentally using various approaches or assays, e.g., flow chamber (Kaplanski et al. 1993; Alon et al. 1995; Finger et al. 1996; Yago et al. 2007; Paschall et al. 2008), biomembrane force probe (BFP) (Evans et al. 2001; Evans and Ritchie 1997), atom force microscopy (AFM) (Fritz et al. 1998; Merkel et al. 1999; Marshall et al. 2005; Lü et al. 2006), micropipette aspiration (Chesla et al. 1998; Long et al. 2001; Shao and Xu 2002), optical tweezers (Kulin et al. 2002; Rinko et al. 2004), fluorescence recovery after photobleaching (FRAP) (Dustin et al. 1996; Tolentino et al. 2008). Only a few works, however, were focused on quantifying $2 \mathrm{D}$ association kinetics mainly due to theoretical and 
technical limitations. For example, association rate (in $\mathrm{s}^{-1}$ ) of homotypic [e.g., cadherin-cadherin (Pierres et al. 1998)] or heterotypic [e.g., E-selectin-ligand (Kaplanski et al. 1993) or CD2-CD48 (Pierres et al. 1997)] molecular pair, defined as the product of 2D forward rate $k_{\mathrm{f}}$ (in $\mu \mathrm{m}^{2} / \mathrm{s}$ ) and site density (in $\mu \mathrm{m}^{-2}$ ), was extracted from the dependence of binding frequency on the distance between apposed surfaces in a flow chamber assay. 2D effective forward rate (in $\mu \mathrm{m}^{4} / \mathrm{s}$ ), the product of $2 \mathrm{D}$ forward rate and contact area (in $\mu \mathrm{m}^{2}$ ), could also be estimated from the dependence of adhesion probability on contact duration as well as site densities of interacting molecules in an adhesion frequency assay (Chesla et al. 1998; Huang et al. 2004; Long et al. 2001; Wu et al. 2007), and from the waiting time distribution of intermittent binding and unbinding events in a biomembrane force probe (BFP) assay (Chen et al. 2008). Surface-bound bond formation is determined by $2 \mathrm{D}$ forward rate of interacting molecules, $k_{\mathrm{f}}$, the association rate per unit contact area per molecule $\left(\mu \mathrm{m}^{2} / \mathrm{s}\right)$. It has long been assumed that $k_{\mathrm{f}}$ is a constant although this has not been tested experimentally and may not be true (Zhu 2000). Visualizing of the bond formation requires simultaneous manipulation of the surface-to-surface approach and separation, and determining of the moments when the bond starts to form or dissociate. It is still unknown, however, how physical factors regulates the surface-bound association kinetics or $2 \mathrm{D}$ forward rate $k_{\mathrm{f}}$.

Regulating factors for bond formation are more complicated as compared to those for bond dissociation. For example, contribution of cellular deformability to flowinduced cell adhesion was assumed to play an important role in stable, shear-resistant rolling (Yago et al. 2002). Enhancing carrier stiffness and roughing carrier surface reduced effective affinity of receptor-ligand interactions (Williams et al. 2001; Wu et al. 2007). Shortening the distance (Pierres et al. 1997) and increasing the relative motion (Chang and Hammer 1999) between two apposed surfaces enhanced association rate under convective flow. Carrier diffusion in shear flow near a wall was also proposed to affect binding rates between surface-bound molecules (Pierres et al. 2001). Regardless of the aforementioned evidences, the underlying carrier-diffusion mechanism, that affects 2D bond formation, still remains little known.

Flow-enhanced cell adhesion is a counter-intuitive phenomenon that has been observed in several biological systems. Three possible transport mechanisms have been proposed: shear-induced sliding of molecular carrier, Brownian motion of carrier, and molecular diffusion (Yago et al. 2007). In a flow chamber assay, the impact of carrier diffusion is coupled with that of carrier sliding, which is hard to be isolated from each other. To exclude the impact of shear-induced sliding of carrier, several approaches that consist in monitoring thermal fluctuations of decorated beads by using weak force probes such as optical trap (Kuo and Sheetz 1993; Svoboda et al. 1993; Veigel et al. 2003), glass microneedles (Ishijima et al. 1994), and BFP (Evans et al. 2001) were used to detect the bindings between single molecular pair (Chen et al. 2008; Kulin et al. 2002; Veigel et al. 1998). Two surfaces bearing respective receptors and ligands were manipulated and thermal fluctuation enables the occurrence of receptor-ligand binding and unbinding. However, contribution of carrier diffusion to 2D forward rate has not been well described.

Here, we developed an alternative thermal fluctuation approach using optical trap set-up, where one of two surfaces was allowed to diffuse in a weakly damped pattern and diffusivity of carrier was regulated using varied laser power (or trap stiffness) to confine the diffusive displacement. Three selectin constructs and their PSGL-1 ligand, which are important cellular adhesive molecules in such biological processes as inflammatory responses, platelet thrombosis, as well as tumor metastasis (Ley 2003), were used to detect the dependence of bond formation on carrier diffusion. Our results indicated that enhancing diffusivity of carrier reduced 2D forward rate and that bond formation between two apposed surfaces was history-dependent.

\section{Materials and methods}

\section{Proteins and antibodies}

Soluble L-selectin (sLs), P-selectin (sPs) or PLE consisting of Lec-EGF domains plus two, nine or no consensus repeats (CRs), and non-blocking anti-sLs (CA21), anti-sPs (S12) (Geng et al. 1990), and anti-PLE (1478) monoclonal antibodies (mAbs) (Mehta et al. 1997), as well as antiPSGL-1 mAb (PL2) (all mouse IgG1, mIgG1) were generous gifts from Dr. Rodger P. McEver (Oklahoma Medical Research Foundation). PSGL-1 constructs were purified following a modified protocol previously reported (Moore et al. 1994). FITC-conjugated goat anti-mouse secondary mAbs and bovine serum albumin (BSA) were purchased from Sigma (St. Louis, MO).

Protein immobilization on microbeads

$2.32 \mu \mathrm{m}$ - and $5.66 \mu \mathrm{m}$-diameter silica microbeads (Bangs, Fishers, IN) were washed in phosphate buffer solution (PBS) (Hyclone, Fishers, IN) for three times, and were incubated overnight at $4{ }^{\circ} \mathrm{C}$ in $5-20 \mu \mathrm{g} / \mathrm{ml}$ of capturing mAbs CA21, S12 or 1478, and PL2, respectively. After rinsing in PBS, the microbeads were sealed with $2 \%$ BSA to block nonspecific adhesion for $8 \mathrm{~h}$. CA21-, S12- or 1478-, and PL2-coated microbeads were then functionalized by incubation in $20 \mathrm{ng} / \mathrm{ml} \mathrm{sLs}$ or sPs or PLE and PSGL-1, respectively, for 
over $12 \mathrm{~h}$ at $4^{\circ} \mathrm{C}$. After rinsing in PBS and being re-blocked with BSA, sLs-, sPs- or PLE-, and PSGL-1-coupled microbeads were ready for thermal fluctuation measurements within 3 days (Fig. 1a). Microbeads coated with BSA and CA21, S12, 1478 or PL2 alone (all blocked with BSA) were used as control. Here, a covalent linkage protocol is no longer used since the simple procedure of physical
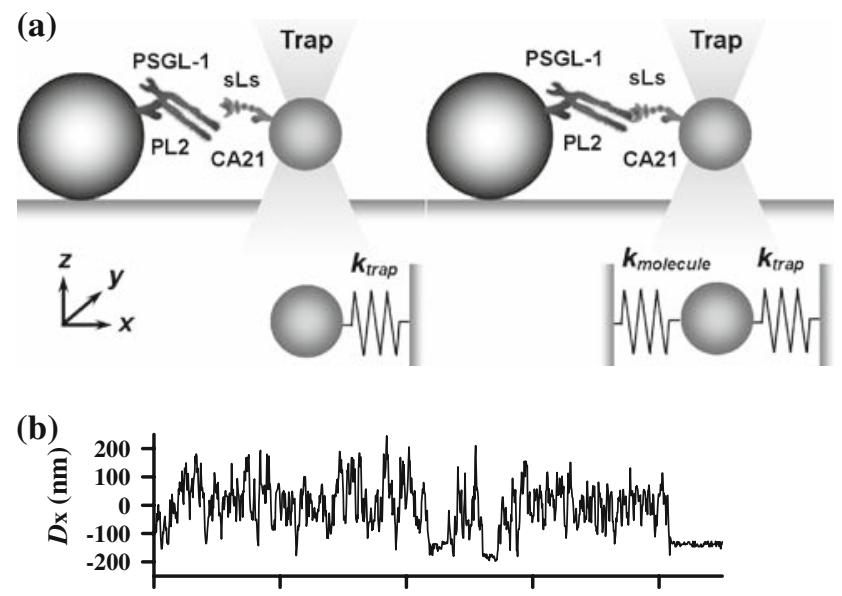

(c)

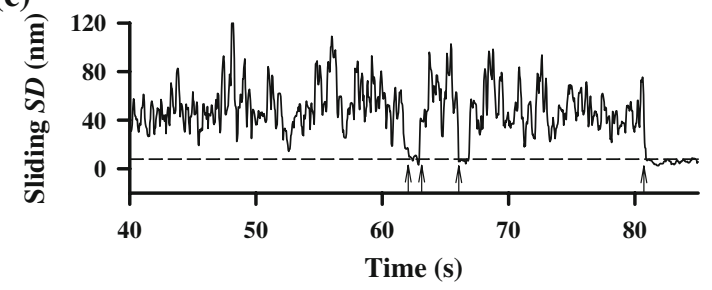

(d)

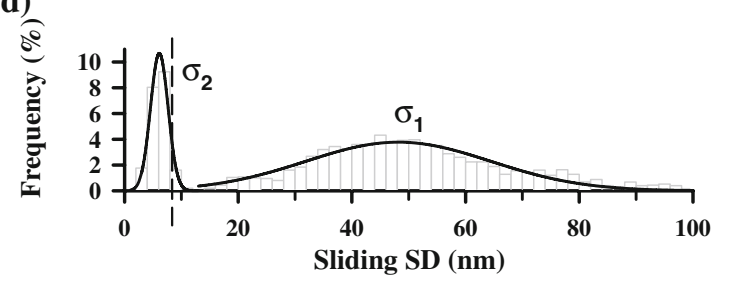

Fig. 1 a Schematic of thermal fluctuation approach using optical trap (not in scale). A $5.66 \mu$ m-diameter microbead coupled with PSGL-1 was fixed on the coverslip and a $2.32 \mu \mathrm{m}$-diameter microbead coupled with selectin (sLs, sPs, or PLE) was trapped in a soft trap with probe stiffness $\sim 10^{-3} \mathrm{pN} / \mathrm{nm}$. Selectin-coupled microbead was fluctuating at the vicinity to PSGL-1-coupled microbead, and stopped moving when bond formation occurred. Once bound, two interacting molecules was physically linked and behaved mechanically like a particle connected with two springs in parallel. b Time course of displacement of selectin-coupled microbead along $x$-axis $\left(D_{x}\right)$ on focus plane (as seen in Supplemental video). Data at $t<40 \mathrm{~s}$ were not presented for the sake of clarity. $\mathbf{c}$ Time course of sliding standard deviation (SD) of $D_{x}$ over a window size of ten frames. Dashed line illustrates the threshold of sliding SD to identify binding state and arrows indicate four binding events. d Histogram of sliding SD corresponding to the data in (c). Two peaks represented unbinding $\left(\sigma_{1}\right)$ and binding $\left(\sigma_{2}\right)$ states, respectively. Dashed line shows same threshold as in (c) absorption via capturing mAbs was found to work well in the current measurements.

Site density determination

Site densities of surface proteins coated on silica microbeads were determined using flow cytometry and immunoradiometric assay (Huang et al. 2004; Ushiyama et al. 1993). To measure densities of S12 and PL2 so adsorbed, for example, one set of S12- or PL2-coated microbeads were incubated with FITC-conjugated goat anti-mouse mAbs at a concentration of $10 \mu \mathrm{g} / \mathrm{ml}$ in $400 \mu \mathrm{l}$ PBS on ice for $40 \mathrm{~min}$. After washing, the microbeads were analyzed by flow cytometer (BD Biosciences, San Jose, CA). Site densities were then calculated by comparing the fluorescence intensities of the silica microbeads with those of standard beads (Bangs, Fishers, IN) (Chesla et al. 1998). Another set of S12- or PL2coated microbeads was incubated with corresponding sPs or PSGL-1 and site density of sPs or PSGL-1 was measured by immunoradiometric assay in an independent measurement (Ushiyama et al. 1993). Thus, a calibration curve was obtained for each protein by plotting the protein site density against the mean fluorescence intensity of the capturing $\mathrm{mAb}$ (data not shown), thereby allowing calculation of the site densities of selectin or PSGL-1 from the mean fluorescence intensities of the capturing mAb.

Optical trap set-up

Each $10^{3}$ selectin- (sLs, sPs, or PLE) and PSGL-1-coupled microbeads were mixed just before injecting into a customer-made glass sample cell $(\sim 14 \times 10 \times 0.5 \mathrm{~cm})$. $5.66 \mu \mathrm{m}$-diameter microbeads coupled with PSGL-1 settled quickly down and adhered stably onto the surface of coverslip substrate. Floating $2.32 \mu \mathrm{m}$-diameter microbeads bearing selectins were captured by a mobile trap (PALM, Zeiss, Germany) and were driven to move slowly to the vicinity of PSGL-1-coated microbead where the initial central distance of two microbeads was pre-set to $\sim 4 \mu \mathrm{m}$. The probe stiffness was as low level as $\sim 10^{-3} \mathrm{pN} / \mathrm{nm}$, which enables the microbead to fluctuate in a weakly damped pattern. Time course of displacement of the microbead due to thermal fluctuation was recorded at 25 frames per second (fps) until stable adhesion was observed (Fig. 1b). After pulling away from the contact to separate two surfaces, the microbead was driven back to enable the occurrence of sequential binding-unbinding events. Such test cycle was repeated $\sim 10$ times for each pair of microbeads and total $>10$ pairs were used in each condition. Adhesion events due to receptor-ligand bond formation were identified by visualizing the sudden stop or sharp reduction of thermal fluctuation. All measurements were done at room temperature $\left(24-28^{\circ} \mathrm{C}\right)$. 
Data analysis

\section{Threshold to identify bond formation}

A cross-correlation method described previously (Gelles et al. 1988) was used to determine the position (or displacement) of $2.32 \mu \mathrm{m}$-diameter microbead with an accuracy of $\sim 2 \mathrm{~nm}$ in $x-y$ plane. Sliding standard deviation (SD) of position was calculated over sequential ten frames (Fig. 1c) and then analyzed using sliding SD histogram to identify binding state $\left(\sigma_{2}\right)$ from unbinding state $\left(\sigma_{1}\right)$ (Chen et al. 2008). Most probable frequency of $\sigma_{2}$ from an ensemble of records $(n>100)$ under same condition was defined as the threshold to visualize bond formation event from thermal fluctuation (Fig. 1d).

\section{Central distance and contact area between two microbeads}

The distance between centers of two microbeads, $d$, was estimated from projected image of two microbeads on focus plane. Upon mirror-symmetry analysis, a window containing brim of microbeads image was selected and the matrix of image window was translated along $x$ - and $y$-axis to search the most correlated position (Gelles et al. 1988). The distance was then calculated from center coordinates of two microbeads. Contact area, $A_{\mathrm{c}}$, which is a function of distance $d$ and molecular length, $l$, follows $A_{\mathrm{c}}=2 \pi r_{2}\left(r_{1}+r_{2}+l-d\right)$ (Rinko et al. 2004), where $r_{1}(=2.83 \mu \mathrm{m})$ and $r_{2}(=1.16 \mu \mathrm{m})$ are radii of two microbeads, and $l$ is the sum of lengths of two capturing mAbs ( $\sim 5 \mathrm{~nm}$ each), PSGL-1 [ $\sim 50 \mathrm{~nm}$ (Li et al. 1996)], and selectin [ $\sim 12,38$, and $4 \mathrm{~nm}$ for sLs, sPs, and PLE constructs, respectively (Ushiyama et al. 1993)] constructs. Since their binding sites locate at respective CR6, CR2, and EGF domain for CA21-sLs, S12-sPs, and 1478-PLE linkage, $l$ yields 72, 87, and $64 \mathrm{~nm}$ for CA21sLs-PSGL-1-PL2, S12-sPs-PSGL-1-PL2, and 1478-PLEPSGL-1-PL2 complex, respectively.

\section{$2 D$ forward rate determination}

Time interval between two sequential bond formation events is the waiting time, $t_{\mathrm{f}}$. As exemplified in Fig. $2 \mathrm{a}$, the cumulative frequency of waiting time $t_{\mathrm{f}}$ for sLs-PSGL-1 bond formation appears to follow an exponential distribution (open circles in Fig. 2a). This enables to predict 2D forward rate upon first order association kinetics,

$\mathrm{d} P_{\mathrm{a}}\left(t_{\mathrm{f}}\right) / d t_{\mathrm{f}}=-A_{\mathrm{c}}\left(t_{\mathrm{f}}\right) m_{\mathrm{r}} m_{\mathrm{l}} k_{\mathrm{f}}\left[1-p_{\mathrm{a}}\left(t_{\mathrm{f}}\right)\right]$,

where $p_{\mathrm{a}}\left(t_{\mathrm{f}}\right)$ is the probability having a bond at time no less than $t_{\mathrm{f}}\left(t \geq t_{\mathrm{f}}\right), A_{\mathrm{c}}\left(t_{\mathrm{f}}\right)$ is the contact area at $t=t_{\mathrm{f}}$, and $m_{\mathrm{r}}$ and $m_{1}$ are the site densities of sLs and PSGL-1, respectively. Noting that $P_{\mathrm{a}}=0$ at $t_{\mathrm{f}}=0$ and that $\overline{A_{\mathrm{c}}}$ is the mean contact area since $A_{\mathrm{c}}$ varies in $\left[0, t_{\mathrm{f}}\right]$, it follows, (a)

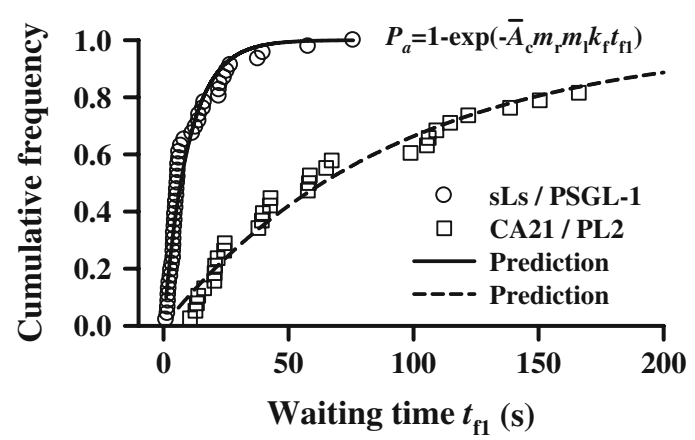

(b)

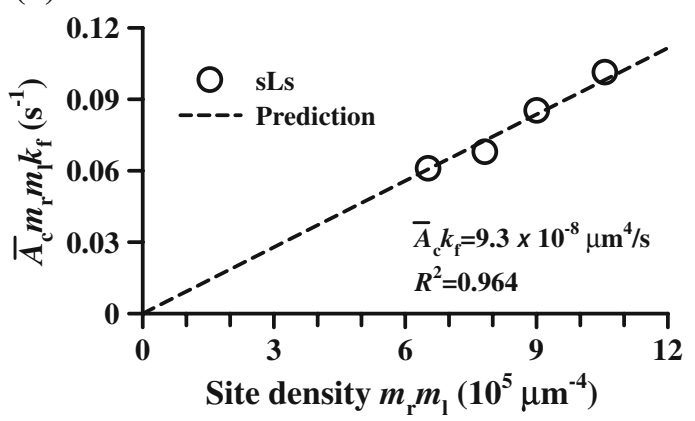

Fig. 2 a Cumulative distribution of $t_{\mathrm{f} 1}$ for specific (open circles) and non-specific (open squares) interactions. Solid and dashed lines indicate respective predictions fitted using Eq. 2. b Dependence of 2D cellular forward rate $\bar{A}_{\mathrm{c}} m_{\mathrm{r}} m_{\mathrm{l}} k_{\mathrm{f}}$ on site densities $m_{\mathrm{r}} \times m_{1}=(6.54$, $7.83,9.02,10.58) \times 10^{5} \mu \mathrm{m}^{-4}$ (open circles) for sLs-PSGL-1 interactions. The slope of fitted line (dashed line) demonstrates 2D effective forward rate $\overline{A_{\mathrm{c}}} k_{\mathrm{f}}$

$p_{\mathrm{a}}\left(t_{\mathrm{f}}\right)=1-\exp \left[-\bar{A}_{\mathrm{c}} m_{\mathrm{r}} m_{\mathrm{l}} k_{\mathrm{f}} t_{\mathrm{f}}\right]$.

$2 \mathrm{D}$ cellular forward rate, $\overline{A_{\mathrm{c}}} m_{\mathrm{r}} m_{\mathrm{l}} k_{\mathrm{f}}$, was then predicted from best-fitting the $p_{\mathrm{a}}\left(t_{\mathrm{f}}\right)$ versus $t_{\mathrm{f}}$ curve to Eq. 2 (solid line in Fig. 2a).

\section{Diffusivity of microbead in optical trap}

Probe stiffness, $k^{\text {trap }}$, was calibrated using energy equipartition theorem and Stokes method (Neuman and Block 2004). Fluctuation of microbeads in a weak trap behaves like a damped diffusion, which can be simplified as free Brownian motion within an infinitely high square well potential (Kusumi et al. 1993). 2D mean square displacement (MSD) at time interval between two consecutive frames, $\delta t$ (40 $\mathrm{ms}$ in the current work), follows

$$
\begin{gathered}
\operatorname{MSD}(n \delta t)=\sum_{j=1}^{N-n-1}\left\{[x(j \delta t+n \delta t)-x(j \delta t)]^{2}\right. \\
\left.+[y(j \delta t+n \delta t)-y(j \delta t)]^{2}\right\} /(N-n-1),
\end{gathered}
$$

where $[x(j \delta t+n \delta t), y(j \delta t+n \delta t)]$ is the coordinates of microbead after spanning a time interval $\Delta t_{n}=n \delta t$ from a starting point $[x(j \delta t),(y(j \delta t)], N$ is the total number of 
frames recorded, and $n$ and $j$ are positive integers where $n$ determines the time increment. In the current work, diffusive coefficient $D$ was determined by fitting MSD at $2 \delta t$, $3 \delta t$, and $4 \delta t$ using a straight line (Kusumi et al. 1993), and the slope of fitted line is defined as $D$. Note that the diffusive coefficient is governed by laser power (or trap stiffness) for the same microbead in the same liquid environment in the current work.

\section{Results}

Binding is specifically mediated by selectin-PSGL-1 interaction

Transient bond formation and dissociation between two microbeads were quantified by thermal fluctuation of $2.32 \mu \mathrm{m}$-diameter microbead at probe stiffness $k^{\text {trap }}=$ $3.6 \times 10^{-3} \mathrm{pN} / \mathrm{nm}$. Time course of microbead displacement was recorded continuously and presented as the dependence of $x$-axis displacement, $D_{\mathrm{x}}$, on time intervals of waiting time $\left(t_{\mathrm{f}}\right)$ and bond lifetime (Fig. 1c). It was obvious that the cumulative frequency of $t_{\mathrm{f}}$ was enhanced much faster for the binding between sLs- and PSGL-1-coupled microbeads than that between two microbeads where only non-blocking mAbs CA21 and PL-2 were present (Fig. 2a), suggesting that the specific binding could be isolated from the non-specific binding. Similar results were obtained for sPs- or PLE-PSGL-1 interactions (data not shown). Such binding specificity was also confirmed by an adhesion frequency measurement where the adhesion was observed when selectin and PSGL-1 were present but abolished when selectin and/or PSGL-1 were absent or when calcium chelator EDTA was present (data not shown). Taken together, the adhesion observed was specifically mediated by selectin-PSGL-1 binding.

\section{D forward rate is determined from first bond formation}

Displacement of sLs-coupled microbead, $D_{x}$, exhibited sequentially a high-magnitude fluctuation phase followed by a low-magnitude fluctuation phase, demonstrating the unbinding and binding states, respectively (Fig. 1b). Sliding SD analysis was employed to identify the two discrete phases, in which sliding SD histogram was found to show two peaks corresponding to the unbinding state $\left(\sigma_{1}\right)$ and binding state $\left(\sigma_{2}\right)$, respectively (Fig. 1d). Individual bond formation events were recognized by determining the starting and ending moments of bond formation occurrence (arrows in Fig. 1c), the time interval of which is the bond lifetime, $t_{\mathrm{r}}$.

Individual first waiting time, $t_{\mathrm{f} 1}$, in each consecutive time course, was pooled together to obtain $t_{\mathrm{f} 1}$ spectrum for first bond formation, since it is most likely that the latter binding event might be affected by the former binding events (seen in the "Discussions"). In the current work, $t_{\mathrm{f}}$ means $t_{\mathrm{f} 1}$ if not specified otherwise. The $p_{\mathrm{a}}\left(t_{\mathrm{f}}\right)$ versus $t_{\mathrm{f}}$ curve for CA21-PL2 interaction (open squares in Fig. 2a) was found to be much lower as compared to that for sLs-PSGL-1 interaction, but still able to be fitted using $P_{\mathrm{a}}\left(t_{\mathrm{f}}\right)=1-\exp \left[-b t_{\mathrm{f}}\right]$ (dashed line in Fig. 2a). Here, $b$ yielded $\sim 0.01$ for CA21-PL2 pair and $\sim 0.04$ for both S12-PL2 and 1478-PL2 pairs, which was one order-ofmagnitude lower than and subtracted from $\bar{A}_{\mathrm{c}} m_{\mathrm{r}} m_{\mathrm{l}} k_{\mathrm{f}}$. This further imparts the confidence that the impact of nonspecific on estimating $2 \mathrm{D}$ forward rate is negligible.

Three selectin constructs, sLs, sPs, and PLE, were used to validate the approach. To test the dependence of bond formation on site densities, four systematically varied site densities of sLs and PSGL- 1 constructs, $m_{\mathrm{r}} \times m_{1}=(6.54$, $7.83,9.02$, and 10.58) $\times 10^{5} \mu \mathrm{m}^{-4}$, were used to measure $t_{\mathrm{f}}$ distribution at each site density. Our data indicated that $\overline{A_{\mathrm{c}}} m_{\mathrm{r}} m_{\mathrm{l}} k_{\mathrm{f}}$ was linearly proportional to site densities, $m_{\mathrm{r}} \times m_{1}$, as expected (open circles in Fig. 2b). The slope of linearly fitted line (dashed line) is termed as 2D effective forward rate $\bar{A}_{\mathrm{c}} k_{\mathrm{f}}$ of sLs-PSGL-1 binding, which measures $9.3 \times 10^{-8} \mu \mathrm{m}^{4} / \mathrm{s}$ (open bar in Fig. 3). Similarly, both P-selectin constructs at $m_{\mathrm{r}} \times m_{1}=(2.11$ and 8.27$) \times$ $10^{5} \mu^{-4}$ (sPs) and at $m_{\mathrm{r}} \times m_{1}=4.13 \times 10^{6} \mu \mathrm{m}^{-4}$ (PLE) were also used to quantify bond association kinetics. 2D effective forward rate $\bar{A}_{\mathrm{c}} k_{\mathrm{f}}$ obtained for sPs-PSGL-1 binding $\left(16.4 \times 10^{-8} \mu \mathrm{m}^{4} \mathrm{~s}^{-1}\right)$ (hatched bar in Fig. 3) was $\sim$ twofold higher than that for sLs-PSGL-1 binding, and one order-of-magnitude higher than that for PLE-PSGL-1 binding $\left(1.3 \times 10^{-8} \mu \mathrm{m}^{4} / \mathrm{s}\right)$ (solid bar in Fig. 3). It was also indicated that 2D forward rate of P-selectin was enhanced by extending extracellular domains over the membrane, as found in the previous work (Huang et al. 2004).

\section{Bond formation is regulated by carrier diffusivity}

It was also found from thermal fluctuation measurements that waiting time $t_{\mathrm{f} 1}$ was linearly correlated with most

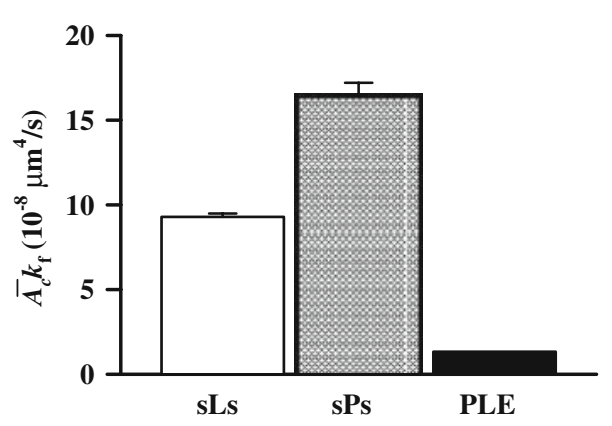

Fig. 32 D effective forward rate $\overline{A_{\mathrm{c}}} k_{\mathrm{f}}$ for sLs-, sPs-, and PLE-PSGL-1 interactions. Data were presented as the mean \pm standard errors (SE) 
probable value of sliding SD for unbinding state $\left(\sigma_{1}\right)$ (Fig. 4a). Since $\sigma_{1}$ characterizes fluctuation of microbeads during unbinding state and $t_{\mathrm{f} 1}$ is correlated to $2 \mathrm{D}$ forward rate, it is reasonable to assume that bond formation observed in thermal fluctuation is regulated by diffusivity of microbeads. To test the hypothesis, laser power was varied to modify the trap stiffness and the impact of diffusive coefficient $D$ on $\overline{A_{\mathrm{c}}} k_{\mathrm{f}}$ was quantified. In a typical measurement, three diffusive coefficients 2,437, 808, and $172 \mathrm{~nm}^{2} / \mathrm{s}$ [corresponding to the trap stiffness $k^{\text {trap }}=(2.1$, 3.6 , and 8.7$\left.) \times 10^{-3} \mathrm{pN} / \mathrm{nm}\right)$ ] were used for visualizing sLs-PSGL-1 bond formation, and 2D effective forward rate so estimated yielded $(1.8,7.9$, and 17.9$) \times 10^{-8} \mu \mathrm{m}^{4} / \mathrm{s}$,
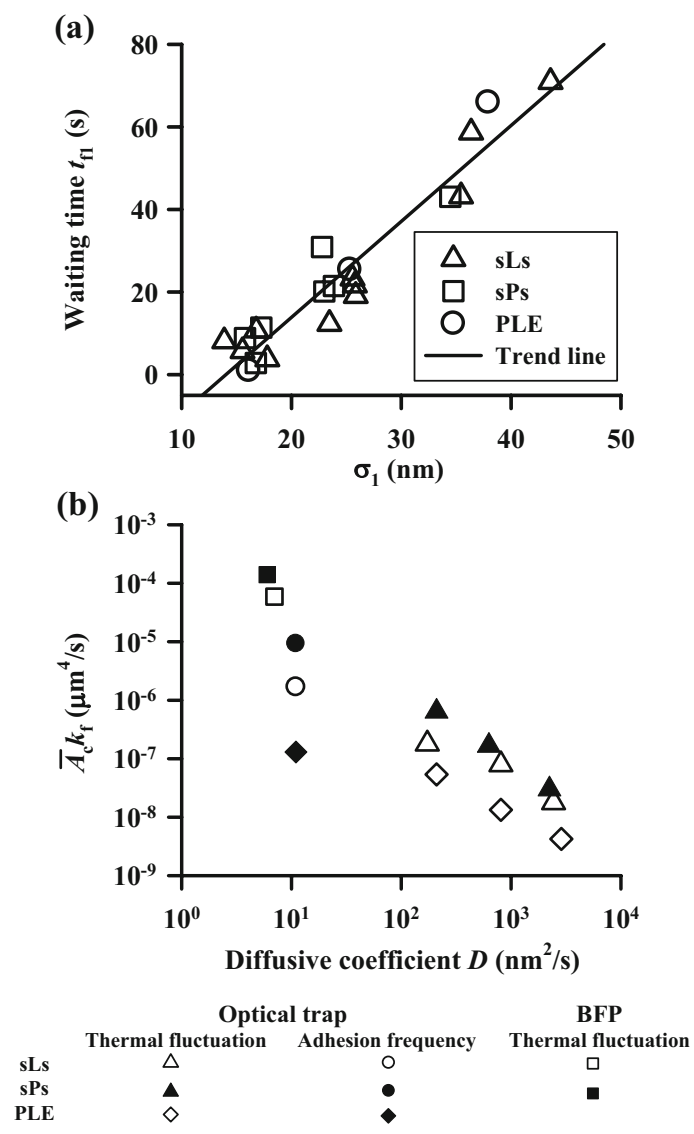

Fig. 4 a Dependence of waiting time $t_{\mathrm{f} 1}$ on most probable sliding SD $\sigma_{1}$ for sLs- (open triangles), sPs- (open squares), and PLE-PSGL-1 (open circles) interactions. Data at three different values of diffusive coefficient, as in (b), were pooled together for each selectin construct. The line is a trend line. b Dependence of 2D effective forward rate $A_{\mathrm{c}} k_{\mathrm{f}}$ on diffusive coefficient $k^{\text {trap }}$ using both thermal fluctuation and adhesion frequency approaches by two techniques of optical trap and BFP for sLs- (open triangles, circle, and square), sPs- (closed triangles, circle, and square), and PLE-PSGL-1 (open diamonds, and closed diamond) interactions. Data for optical trap assay were obtained from the current study, and presented without error bars for the sake of clarity. Data for BFP assay were adopted from (Chen et al. 2008) and diffusive coefficient was estimated based on published sliding SD histogram respectively. This turned out to be a linear increase of $\overline{A_{\mathrm{c}}} k_{\mathrm{f}}$ with $\log$ arithm of diffusive coefficient, $\log (D)$ (open triangles in Fig. 4b).

To further test the hypothesis, diffusive coefficient was set to a low value of $11 \mathrm{~nm}^{2} / \mathrm{s}\left(k^{\text {trap }}=5.6 \times 10^{-2} \mathrm{pN} / \mathrm{nm}\right)$ where thermal fluctuation of selectin-coupled microbead is unable to be well visualized. Thus, thermal fluctuation approach is no longer available since the binding and unbinding states were hard to be identified in the current set-up. Instead, adhesion frequency approach was used to quantify 2D kinetics of selectin-PSGL-1 interactions (Chesla et al. 1998). Briefly, selectin-coupled microbead was driven by piezo translator (PI, Germany), and two microbeads were impinged into contact with a constant contact area, $\hat{A_{\mathrm{c}}}$, at pre-set contact durations $(0.5-10 \mathrm{~s})$. Adhesion event was identified when selectin-coupled microbead bounced back to trap center at the moment of separating two surfaces. Test cycle of approach-contactretract was repeated $\sim 50$ times for each microbead pair, and totally $>3$ pairs of microbeads were used to obtain the dependence of adhesion frequency, $P_{\mathrm{a}}$, on each contact duration, $t$. $2 \mathrm{D}$ cellular binding affinity, $\hat{A_{\mathrm{c}}} m_{\mathrm{r}} m_{\mathrm{l}} K_{\mathrm{a}}^{0}$, and reverse rate, $k_{\mathrm{r}}^{0}$, were predicted by fitting the data using a well-developed model (Chesla et al. 1998),

$P_{a}=1-\exp \left\{-\hat{A}_{\mathrm{c}} m_{r} m_{l} K_{a}^{0}\left[1-\exp \left(-k_{r}^{0} t\right)\right]\right\}$.

Here $K_{\mathrm{a}}^{0}$ is $2 \mathrm{D}$ binding affinity. In a typical sLs-PSGL-1 binding measurement, three binding curves were obtained at

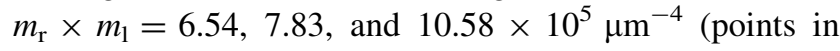
Fig. 5a) and the data fitted well the model (Eq. 4) (lines in Fig. 5a). A set of kinetic parameter $\left(\hat{A_{\mathrm{c}}} m_{\mathrm{r}} m_{\mathrm{l}} K_{\mathrm{a}}^{0}, k_{\mathrm{r}}^{0}\right)$ was obtained from best-fitting one binding curve and three sets of kinetic parameters were averaged at three densities (Fig. 5b). Thus, 2D effective forward rate $\hat{A}_{\mathrm{c}} k_{\mathrm{f}}$, calculated from $\hat{A}_{\mathrm{c}} k_{\mathrm{f}}=\left(\hat{A}_{\mathrm{c}} m_{\mathrm{r}} m_{\mathrm{l}} K_{\mathrm{a}}^{0}\right) \times k_{\mathrm{r}}^{0} /\left(m_{\mathrm{r}} \times m_{\mathrm{l}}\right)$, yielded $1.7 \times$ $10^{-6} \mu \mathrm{m}^{4} / \mathrm{s}$. Considering that the constant contact area $\hat{A_{\mathrm{c}}}$ in adhesion frequency assay $\left(\sim 0.5 \mu \mathrm{m}^{2}\right)$ is comparable to the averaged value in thermal fluctuation assay $\left(\sim 0.8 \mu \mathrm{m}^{2}\right)$, $2 \mathrm{D}$ effective forward rate so obtained from adhesion frequency measurements at low $D=11 \mathrm{~nm}^{2} / \mathrm{s}$ (open circle in Fig. 4b) was one or two order-of-magnitude higher than those obtained from thermal fluctuation measurements at high $D=2,437,808$, and $172 \mathrm{~nm}^{2} / \mathrm{s}$. This can also been seen from thermal fluctuation measurements using BFP technique where $\hat{A}_{\mathrm{c}} k_{\mathrm{f}}$ yielded a high value of $5.9 \times$ $10^{-5} \mu \mathrm{m}^{4} / \mathrm{s}$ at low $D \sim 7 \mathrm{~nm}^{2} / \mathrm{s}$ (open square in Fig. $4 \mathrm{~b}$ ). Here, $D$ was estimated using the calibrating curve between $D$ and $\sigma_{1}$ (data not shown), and $\sigma_{1}=5.3 \mathrm{~nm}$ was adapted from the literature (Chen et al. 2008). Similar dependence of $\hat{A}_{\mathrm{c}} k_{\mathrm{f}}$ on $D$ was also found for sPs-PSGL-1 (solid triangles, circle, and square) and for PLE-PSGL-1 (open and solid diamonds) interactions (Fig. 4b). Taken together, 2D effective forward rate $A_{\mathrm{c}} k_{\mathrm{f}}$ was found to decrease with 
(a)
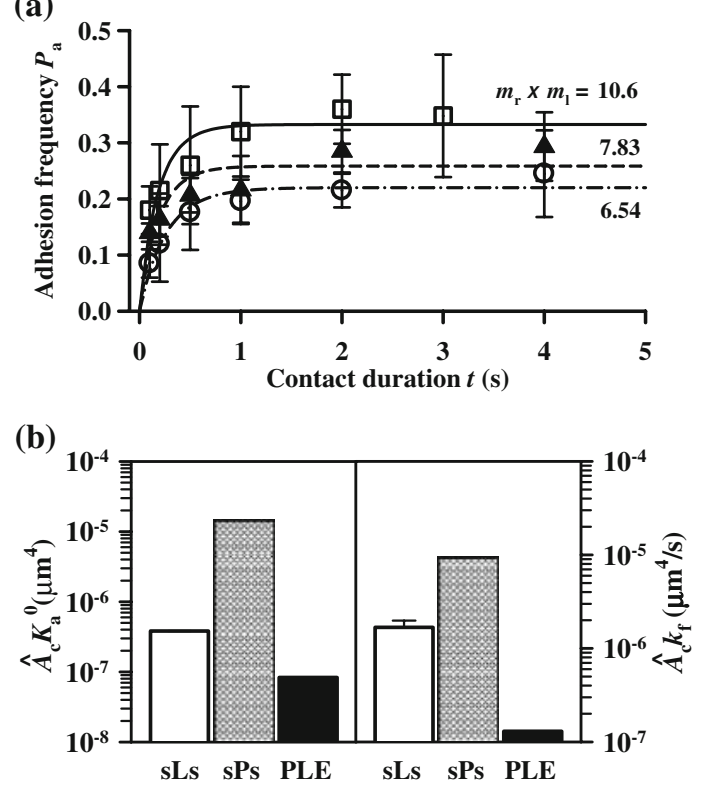

Fig. 5 a Dependence of adhesion frequency on contact duration at $m_{\mathrm{r}} \times m_{1}=6.54$ (open circles), 7.83 (closed triangles), $10.6 \times 10^{5}$ (open squares) $\mu \mathrm{m}^{-4}$ for sLs-PSGL-1 interactions measured using adhesion frequency approach. Data (points), obtained from 3 to 6 microbead pairs and presented as the mean \pm SD at each contact time, were compared to the predictions (lines) fitted using Eq. 3. b 2D effective binding affinity $\hat{A}_{\mathrm{c}} K_{\mathrm{a}}^{0}$ and forward rate $\hat{A}_{\mathrm{c}} k_{f}$ for sLs- (open bars), sPs- (hatched bars), and PLE-PSGL-1 (solid bars) interactions. Data were presented as the mean \pm SD for sLs-PSGL-1 interactions (note that error bar for $\hat{A}_{\mathrm{c}} k_{\mathrm{f}}$ was too small to be visible). No error bars were presented for sPs- and PLE-PSGL-1 interactions, since only a single site density of $m_{\mathrm{r}} \times m_{1}=2.3 \times 10^{4}$ and $4.1 \times 10^{6} \mu \mathrm{m}^{-4}$ was used, respectively

diffusivity of microbeads, suggesting that microbead fluctuation governs bond formation.

Forward rate increases with binding times

Binding characteristics in consecutive bind-unbinding transitions vary from time to time since receptor-ligand interaction may be binding history-dependent (Zarnitsyna et al. 2007). To further understand the history-dependent kinetics, time courses of thermal fluctuation for $n$th $(n=1$, $2,3, \ldots$ ) binding (or unbinding) events for sLs-PSGL-1 interactions were collected in a consecutive spectrum and the waiting time $t_{\mathrm{f}}$ was normalized using the maximum value in that spectrum. It was found that waiting time $t_{\mathrm{f}}$ decreased as binding-unbinding transition proceeded and it finally reduced to the baseline level one order-of-magnitude lower at seventh binding event than that at first binding event (Fig. 6a). This turned out to be one order-ofmagnitude higher of $2 \mathrm{D}$ effective forward rate at the seventh binding event $\left(14.5 \times 10^{-7} \mu \mathrm{m}^{4} / \mathrm{s}\right)$ as compared to that at the first binding event $\left(0.7 \times 10^{-7} \mu \mathrm{m}^{4} / \mathrm{s}\right.$ ) (open triangles in Fig. 6b). Similar enhancement was also found for sPs- or PLE-PSGL-1 interactions (open squares and circles in Fig. 6b). Thus, these results indicated that adhesion is reinforced from time to time in thermal fluctuation measurements.

\section{Discussion}

The goal of the current study is to characterize the association kinetics of receptor-ligand interactions and to quantify the impact of carrier diffusivity on association kinetics. We developed a thermal fluctuation assay, using optical trap technique, to visualize bond formation of selectin-PSGL-1 interaction. To maximize the unbindingbinding transition so observed, a weak trap with a lower $k^{\text {trap }} \sim 10^{-3} \mathrm{pN} / \mathrm{nm}$ was used, which enables preventing the microbead escaping from the trap but has insignificant impacts on microbead fluctuation. The thermal fluctuation was monitored at a temporal resolution of $25 \mathrm{fps}$ and spatial resolution of $\sim 2 \mathrm{~nm}$. Non-specific $P_{\mathrm{a}}$ was $<5 \%$ (data not shown) as compared to specific $P_{\mathrm{a}} \sim 20-30 \%$ (Fig. 5a) and cumulative frequency of $t_{\mathrm{f}}$ for specific interactions was ultimately distinctive with that for nonspecific bindings (Fig. 2a). The approach not only provides a quantitative measurement in $2 \mathrm{D}$ kinetics of bond formation, but it also enables to quantify the biophysical

(a)

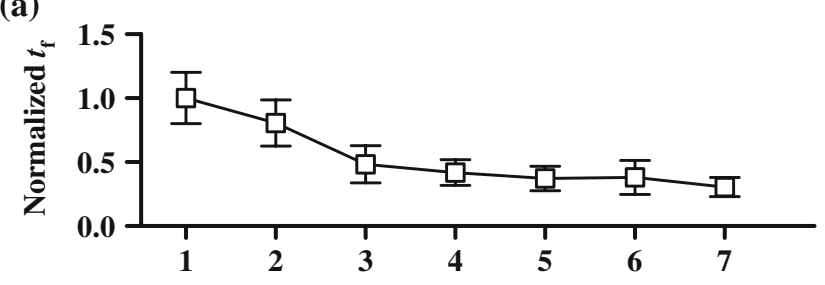

(b)

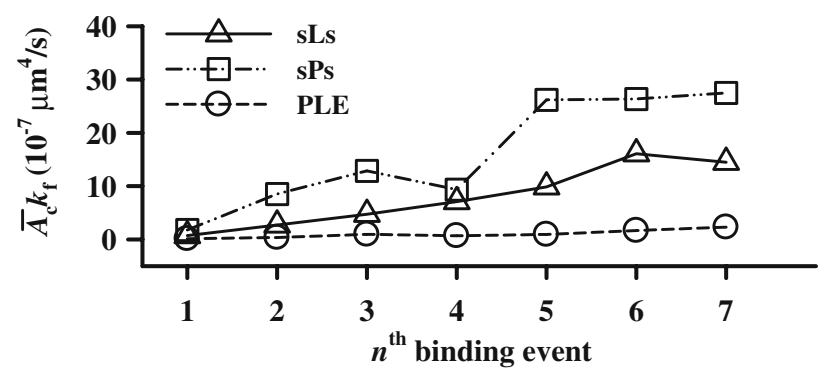

Fig. 6 a Variation of waiting time $t_{\mathrm{f}}$ with consecutive binding event for sLs-PSGL-1 interactions at $m_{\mathrm{r}} \times m_{1}=2.13 \times 10^{5} \mu \mathrm{m}^{-4}$. Data were obtained from normalizing pooled waiting time $t_{\mathrm{f}}$ at $n$th binding event by maximum value at that spectrum and presented as mean $\pm \mathrm{SE}$ (points). b Variation of 2D effective forward rates $\bar{A}_{\mathrm{c}} k_{\mathrm{f}}$ with consecutive $n$th binding event for sLs-, sPs-, and PLE-PSGL-1 interactions at $m_{\mathrm{r}} \times m_{1}=\left(7.83,2.11\right.$, and 41.3) $\times 10^{5} \mu \mathrm{m}^{-4}$, respectively. Data were obtained from fitting pooled waiting time $t_{\mathrm{f}}$ at $n$th binding event using Eq. 2 (points) 
aspects of receptor-ligand interactions, e.g., forced association of bond formation by varying probe stiffness.

Bond formation and dissociation of selectin-ligand interactions occur under blood flow. In an in vitro flow chamber assay, rolling and/or tethering of selectinexpressing cells or microbeads over PSGL-1-immobilized substrate is strongly regulated by shear flow. This turns out to be a complicated association kinetics in which the relative motion between flowing cells and substrate and the cell (or microbead) sedimentation play an important role in manipulating rolling velocity and/or tethering lifetime (Chang and Hammer 1999; Kaplanski et al. 1993; Pierres et al. 1997; Pierres et al. 1998). In addition to the transport of molecular carrier, other factors such as carrier fluctuation and molecular diffusion were found to regulate flowinduced bond formation (Yago et al. 2007). In our optical trap set-up, however, no shear flow is required, and bond formation of selectin-PSGL-1 binding is governed by thermal fluctuation of microbead, molecular diffusion, and intrinsic reaction kinetics. This simplifies the bond association process by ignoring the transport mechanism of sliding velocity. More importantly, such the thermal fluctuation assay enables to isolate the impact of carrier diffusion on association kinetics of surface-bound receptor-ligand interactions.

We first compared 2D association kinetics on varied carrier diffusivity using same set-up (optical trap) but different approaches (thermal fluctuation vs. adhesion frequency). In thermal fluctuation measurement, two surfaces were unable to hold steady contact during time interval $\left[0, t_{\mathrm{f}}\right]$ when selectin-coupled microbead fluctuated instantaneously forward or backward along $x$-axis in a weak trap. Microbead fluctuation reduces the opportunities for two surface-bound molecules to collide with each other, which lowers the effective contact time and prolongs the waiting time to form a bond. In adhesion frequency measurement, however, two surfaces were impinged to make physical contact effectively within entire contact duration $\left[0, t_{\mathrm{f}}\right]$. Microbead fluctuation was significantly reduced and had very less impact on bond formation. This turned out to be one or two order-of-magnitude differences in $2 \mathrm{D}$ effective forward rate $\bar{A}_{\mathrm{c}} k_{\mathrm{f}}$ obtained from two approaches (Figs. 3, 5b), even though follows the same descending order in sPs, sLs, and PLE. This interpretation can be further supported when reducing diffusive coefficient $D$ via enhancing trap stiffness to confine the diffusive displacement in thermal fluctuation measurement, resulting in one or two order-of-magnitude higher $\bar{A}_{\mathrm{c}} k_{\mathrm{f}}$ when $D$ was one order-of-magnitude reduced (Fig. 4b). Similarly, $\bar{A}_{\mathrm{c}} k_{\mathrm{f}}$ so measured using same probe stiffness in BFP set-up were comparable in both approaches (Chen et al. 2008). We further compared 2D association kinetics measurements using same approach (thermal fluctuation) but different techniques [optical trap vs. BFP (Chen et al. 2008)]. Again, three order-of-magnitude difference in $2 \mathrm{D}$ effective forward rate $\left(\sim 10^{-8}\right.$ vs. $\sim 10^{-5} \mu \mathrm{m}^{4} / \mathrm{s}$ for sLs and $\sim 10^{-7}$ vs. $\sim 10^{-4} \mu \mathrm{m}^{4} / \mathrm{s}$ for sPs) might contribute to distinctive nature of microbead fluctuation in optical trap $\left(D \sim 10^{3} \mathrm{~nm}^{2} / \mathrm{s}\right)$ and BFP $\left(D \sim 10^{1} \mathrm{~nm}^{2} / \mathrm{s}\right)$ assays.

This dependence of $2 \mathrm{D}$ forward rate on microbead diffusivity might provide clues to understand $2 \mathrm{D}$ association kinetics from the viewpoint of microbead collision. Intuitively, higher diffusivity seems to enhance the chance of binding because of larger contact zone. 2D bond formation, however, is governed by collision frequency between two surfaces, $H_{\mathrm{p}}$, and averaged fractional contact time per each collision, $\tau_{\mathrm{c}}$ (Yago et al. 2007). Collision frequency and contact time between two surfaces could be estimated from fluid dynamics theory in a flow-induced bond formation approach, which was, in turn, used to predict 2D forward rate (Bell 1981; Evans and Proctor 1978). In a thermal fluctuation measurement, $H_{\mathrm{p}}$ is determined by a distributed band of natural frequency of microbead fluctuation and is able to be characterized by a roll-off frequency $f_{\mathrm{c}}=k^{\text {trap }} /$ $(2 \pi \mu)$ (where $\mu$ is dynamic viscosity of medium), in which $H_{\mathrm{p}}$ increases with $k^{\text {trap }}$. Meanwhile, high $k^{\text {trap }}$ lowers the magnitude of microbead fluctuation and, in turn, reduces $\tau_{\mathrm{c}}$ within contact zone. Thus, the fact that $2 \mathrm{D}$ forward rate is enhanced with probe stiffness implies that $H_{\mathrm{p}}$ dominates bond formation process in thermal fluctuation measurements. This is presumably why $k_{\mathrm{f}}$ was found to be inversely related in the current work but it seems intuitive that $k_{\mathrm{f}}$ should be positively related to the diffusivity of microbead. It should be pointed out that the impact of weakly damped (not freely random) microbead fluctuation and of intermittent contact and no-contact waiting time distribution should be taken into account if one attempts to formulate the equation correlating $\bar{A}_{\mathrm{c}} k_{\mathrm{f}}$ with $D$. Moreover, while the impact of diffusive displacement on microbead diffusivity was quantified in the current work, the effect of other physical factors such as medium viscosity and bead size needs to be quantified in the future investigation.

Separating distance between two microbeads may also affect the diffusivity of microbead and the contact area $A_{\mathrm{c}}$ between two surfaces. In the current work, the central distance is pre-set to $\sim 4 \mu \mathrm{m}$ at the starting point and the initial gap distance between two surfaces was only $0.01 \mu \mathrm{m}$ $(4-5.66 / 2-2.32 / 2=0.01 \mu \mathrm{m})$. Microbead diffusion at low $k^{\text {trap }}$ presents the receptors to counterpart ligands and enables the occurrence of bond formation. Note that the amplitude of thermal fluctuation was $>100 \mathrm{~nm}$ (Fig. 1b), which is ten times higher than gap distance. We also monitored the time course of central distance $d$ frame-byframe during time interval $\left[0, t_{\mathrm{f}}\right]$ and calculated the mean value and its variation for different microbead pairs. No significant difference in initial separation distance was 
found in all cases for sPs, PLE, and sLs binding (data not shown), suggesting that instantaneous contact area $A_{\mathrm{c}}$ be approximately invariable during waiting time $\left[0, t_{\mathrm{f}}\right]$. This is true since the standard deviation of $A_{\mathrm{c}}$ was found to be $2-7 \%$ of mean value, which could be ignored. It should be pointed out that 3D imaging, rather than projected imaging, of microbead fluctuation is required when one attempts to isolate the impact of $A_{\mathrm{c}}$ from the lumped 2D effective forward rate, $\overline{A_{\mathrm{c}}} k_{\mathrm{f}}$.

It is realized that consecutive binding-unbinding events contain the body of information for sequential bond formation and dissociation. Upon the assumption that the binding might be history-dependent, only the data of waiting time for first bond formation were pooled together to predict 2D forward rate in thermal fluctuation approach. This history-dependence was seen in remarkable reduction of waiting time and significant enhancement of 2D effective forward rate in following $n$th ( $n=2,3, \ldots$ ) binding event (Fig. 6a, b). It has long been noticed that shortening the distance reduces dramatically the association rate of two microbeads (Pierres et al. 1997, 1998) and that secondary association is one order-ofmagnitude faster than first association in flow chamber measurement (Kaplanski et al. 1993). We monitored the time course of central distance $d$ and found that it was shortened up to $10-15 \mathrm{~nm}$ from first to seventh binding event, which corresponded to sixfold enhancement in 2D effective forward rate (cf. Fig. 6b). While cell deformability and cell signaling are assumed to be major factors to regulate the history-dependent cell adhesion described previously (Paschall et al. 2008; Zarnitsyna et al. 2007), the line of reasoning for history-dependent association reported here might be attributed to the following aspects. The probability of rebinding is enhanced when two surfaces become closer after the first binding event. The contact area and collision frequency between two surfaces are increased and more molecule pairs have the opportunity to bind. Multiple bonds are formed in the sequential binding events which accelerates the following bond formation. The reduction of gap distance lowers the diffusivity of microbead and/or of receptors. Regardless of that the underlying mechanism remains to be elucidated in future works, our data indicated that the lifetime of selectin-ligand bond was twofold enhanced and the fluctuation magnitude of binding state was reduced to $2 / 5$ for the seventh binding event as compared to first binding event.

Technically, the thermal fluctuation approach developed here requires further improvements to better quantify $2 \mathrm{D}$ binding kinetics of receptor-ligand interactions. For example, low sampling rate at $25 \mathrm{fps}$ is reasonable in quantifying the impact of microbead diffusivity on bond formation but may not be sufficient to track the short-lived events. And since the distance $d$ was measured from a projected image, 3D measurement is required in the future study to enhance the accuracy of calculating diffusive coefficient $D$ and contact area $A_{\mathrm{c}}$. Laser-induced heating is another factor which could affect thermal fluctuation measurements using laser trap. In the current study, the temperature in sample pool under 0.04-0.05 W laser power used was found to exhibit an initial quick increase (in $\sim 1 \mathrm{~min}$ ) before reaching room temperature $\left(24-28^{\circ} \mathrm{C}\right)$ and then to fluctuate within a variation of $\sim 0.2^{\circ} \mathrm{C}$ for $>3 \mathrm{~h}$, as expected (Peterman et al. 2003; Zhang et al. 2008). Even though the effect of temperature increase on 2D forward rate measurement was extremely limited, accurate temperature control is still required to optimize the measurements in further investigations.

Acknowledgments We thank Dr. R. P. McEver for the generous gifts of sPs, S12, sLs, CA21, PLE, 1478 and PL2 proteins, Dr. Z. Z. Ye for PSGL-1 purification. This work was supported by National Natural Science Foundation of China grants 30730032 and 10332060 , National Key Basic Research Foundation of China grant 2006CB910303, National High Technology Research and Development Program of China grant 2007AA02Z306, and Chinese Academy of Sciences grant 2005-1-16 (M.L.).

\section{References}

Albelda SM (1993) Biology of disease-role of integrins and other cell-adhesion molecules in tumor progression and metastasis. Lab Invest 68:4-17

Alon R, Hammer DA, Springer TA (1995) Lifetime of the P-selectincarbohydrate bond and its response to tensile force in hydrodynamic flow. Nature 374:539-542. doi:10.1038/374539a0

Bell GI (1978) Models for the specific adhesion of cells to cells. Science 200:618-627. doi:10.1126/science.347575

Bell GI (1981) Estimate of the sticking probability for cells in uniform shear flow with adhesion caused by specific bonds. Cell Biophys 3:289-304. doi:10.1007/BF02782629

Chang KC, Hammer DA (1999) The forward rate of binding of surface-tethered reactants: effect of relative motion between two surfaces. Biophys J 76:1280-1292. doi:10.1016/S0006-3495(99) 77291-7

Chen S, Alon R, Fuhlbrigge RC, Springer TA (1997) Rolling and transient tethering of leukocytes on antibodies reveal specializations of selectins. Proc Natl Acad Sci USA 94:3172-3177. doi:10.1073/pnas.94.7.3172

Chen W, Evans EA, McEver RP, Zhu C (2008) Monitoring receptorligand interactions between surfaces by thermal fluctuations. Biophys J 94:694-701. doi:10.1529/biophysj.107.117895

Chesla SE, Selvaraj P, Zhu C (1998) Measuring two-dimensional receptor-ligand binding kinetics by micropipette. Biophys $\mathrm{J}$ 75:1553-1572. doi:10.1016/S0006-3495(98)74074-3

Dammer U, Hegner M, Anselmetti D, Wagner P, Dreier M, Huber W et al (1996) Specific antigen/antibody interactions measured by force microscopy. Biophys J 70:2437-2441. doi:10.1016/ S0006-3495(96)79814-4

Dustin ML, Ferguson LM, Chan PY, Springer TA, Golan DE (1996) Visualization of CD2 interaction with LFA-3 and determination of the two-dimensional dissociation constant for adhesion receptors in a contact area. J Cell Biol 132:465-474. doi: 10.1083/jcb.132.3.465 
Evans CW, Proctor J (1978) A collision analysis of lymphoid cell aggregation. J Cell Sci 33:17-36

Evans E, Ritchie K (1997) Dynamic strength of molecular adhesion bonds. Biophys J 72:1541-1555. doi:10.1016/S0006-3495(97) 78802-7

Evans E, Leung A, Hammer D, Simon S (2001) Chemically distinct transition states govern rapid dissociation of single L-selectin bonds under force. Proc Natl Acad Sci USA 98:3784-3789. doi: 10.1073/pnas.061324998

Finger EB, Puri KD, Alon R, Lawrence MB, von Andrian $\mathrm{UH}$, Springer TA (1996) Adhesion through L-selectin requires a threshold hydrodynamic shear. Nature 379:266-269. doi: $10.1038 / 379266 \mathrm{a} 0$

Florin EL, Moy VT, Gaub HE (1994) Adhesion forces between individual ligand-receptor pairs. Science 264:415-417. doi: 10.1126/science. 8153628

Fritz J, Katopodis AG, Kolbinger F, Anselmetti D (1998) Forcemediated kinetics of single P-selectin ligand complexes observed by atomic force microscopy. Proc Natl Acad Sci USA 95:1228312288. doi:10.1073/pnas.95.21.12283

Galkina E, Ley K (2007) Vascular adhesion molecules in atherosclerosis. Arterioscl Throm Vas 27:2292-2301. doi:10.1161/ ATVBAHA.107.149179

Gelles J, Schnapp BJ, Sheetz MP (1988) Tracking kinesin-driven movements with nanometre-scale precision. Nature 331:450453. doi: $10.1038 / 331450 \mathrm{a} 0$

Geng JG, Bevilacqua MP, Moore KL, McIntyre TM, Prescott SM, Kim JM et al (1990) Rapid neutrophil adhesion to activated endothelium mediated by GMP-140. Nature 343:757-760. doi: $10.1038 / 343757 \mathrm{a} 0$

Grinnell F (1992) Wound repair, keratinocyte activation and integrin modulation. J Cell Sci 101:1-5

Hammer DA, Lauffenburger DA (1987) A dynamical model for receptor-mediated cell adhesion to surfaces. Biophys J 52:475487. doi:10.1016/S0006-3495(87)83236-8

Huang J, Chen J, Chesla SE, Yago T, Mehta P, McEver RP et al (2004) Quantifying the effects of molecular orientation and length on two-dimensional receptor-ligand binding kinetics. J Biol Chem 279:44915-44923. doi:10.1074/jbc.M407039200

Ishijima A, Harada Y, Kojima H, Funatsu T, Higuchi H, Yanagida T (1994) Single-molecule analysis of the actomyosin motor using nano-manipulation. Biochem Biophys Res Commun 199:10571063. doi:10.1006/bbrc. 1994.1336

Kaplanski G, Farnarier C, Tissot O, Pierres A, Benoliel AM, Alessi MC et al (1993) Granulocyte endothelium initial adhesionanalysis of transient binding events mediated by E-selectin in a laminar shear-flow. Biophys J 64:1922-1933. doi:10.1016/ S0006-3495(93)81563-7

Kulin S, Kishore R, Hubbard JB, Helmerson K (2002) Real-time measurement of spontaneous antigen-antibody dissociation. Biophys J 83:1965-1973. doi:10.1016/S0006-3495(02)73958-1

Kuo SC, Sheetz MP (1993) Force of single kinesin molecules measured with optical tweezers. Science 260:232-234. doi: 10.1126/science.8469975

Kusumi A, Sako Y, Yamamoto M (1993) Confined lateral diffusion of membrane-receptors as studied by single-particle tracking (Nanovid microscopy)_effects of calcium-induced differentiation in cultured epithelial-cells. Biophys J 65:2021-2040. doi: 10.1016/S0006-3495(93)81253-0

Levin JD, Ting-Beall HP, Hochmuth RM (2001) Correlating the kinetics of cytokine-induced E-selectin adhesion and expression on endothelial cells. Biophys J 80:656-667. doi:10.1016/ S0006-3495(01)76046-8

Ley K (2003) The role of selectins in inflammation and disease. Trends Mol Med 9:263-268. doi:10.1016/S1471-4914(03)00071-6
Li F, Erickson HP, James JA, Moore KL, Cummings RD, McEver RP (1996) Visualization of P-selectin glycoprotein ligand-1 as a highly extended molecule and mapping of protein epitopes for monoclonal antibodies. J Biol Chem 271:6342-6348. doi: $10.1074 /$ jbc.271.11.6342

Long M, Zhao H, Huang KS, Zhu C (2001) Kinetic measurements of cell surface E-selectin/carbohydrate ligand interactions. Ann Biomed Eng 29:935-946. doi:10.1114/1.1415529

Lü SQ, Ye ZY, Zhu C, Long M (2006) Quantifying the effects of contact duration, loading rate, and approach velocity on P-selectin-PSGL-1 interactions using AFM. Polymer (Guildf) 47:2539-2547. doi:10.1016/j.polymer.2005.11.095

Marshall BT, Sarangapani KK, Lou JH, McEver RP, Zhu C (2005) Force history dependence of receptor-ligand dissociation. Biophys J 88:1458-1466. doi:10.1529/biophysj.104.050567

Mehta P, Patel KD, Laue TM, Erickson HP, McEver RP (1997) Soluble monomeric P-selectin containing only the lectin and epidermal growth factor domains binds to P-selectin glycoprotein ligand-1 on leukocytes. Blood 90:2381-2389

Merkel R, Nassoy P, Leung A, Ritchie K, Evans E (1999) Energy landscapes of receptor-ligand bonds explored with dynamic force spectroscopy. Nature 397:50-53. doi:10.1038/16219

Moore KL, Eaton SF, Lyons DE, Lichenstein HS, Cummings RD, McEver RP (1994) The P-selectin glycoprotein ligand from human neutrophils displays sialylated, fucosylated, $O$-linked poly- $N$-acetyllactosamine. J Biol Chem 269:23318-23327

Neuman KC, Block SM (2004) Optical trapping. Rev Sci Instrum 75:2787-2809. doi:10.1063/1.1785844

Paschall CD, Guilford WH, Lawrence MB (2008) Enhancement of L-selectin, but not P-selectin, bond formation frequency by convective flow. Biophys J 94:1034-1045. doi:10.1529/biophysj. 106.098707

Peterman EJG, Gittes F, Schmidt CF (2003) Laser-induced heating in optical traps. Biophys J 84:1308-1316. doi:10.1016/S0006-3495 (03) 74946-7

Pierres A, Benoliel AM, Bongrand P, vander Merwe PA (1997) The dependence of the association rate of surface-attached adhesion molecules CD2 and CD48 on separation distance. FEBS Lett 403:239-244. doi:10.1016/S0014-5793(97)00060-4

Pierres A, Feracci H, Delmas V, Benoliel AM, Thiery JP, Bongrand P (1998) Experimental study of the interaction range and association rate of surface-attached cadherin 11. Proc Natl Acad Sci USA 95:9256-9261. doi:10.1073/pnas.95.16.9256

Pierres A, Benoliel AM, Zhu C, Bongrand P (2001) Diffusion of microspheres in shear flow near a wall: use to measure binding rates between attached molecules. Biophys J 81:25-42. doi: 10.1016/S0006-3495(01)75677-9

Rinko LJ, Lawrence MB, Guilford WH (2004) The molecular mechanics of P- and L-selectin lectin domains binding to PSGL-1. Biophys J 86:544-554. doi:10.1016/S0006-3495(04)74133-8

Shao JY, Xu JB (2002) A modified micropipette aspiration technique and its application to tether formation from human neutrophils. J Biomech Eng-T ASME 124:388-396. doi:10.1115/1.1486469

Springer TA (1995) Traffic signals on endothelium for lymphocyte recirculation and leukocyte emigration. Annu Rev Physiol 57:827-872. doi:10.1146/annurev.ph.57.030195.004143

Svoboda K, Schmidt CF, Schnapp BJ, Block SM (1993) Direct observation of kinesin stepping by optical trapping interferometry. Nature 365:721-727. doi:10.1038/365721a0

Tees DFJ, Woodward JT, Hammer DA (2001) Reliability theory for receptor-ligand bond dissociation. J Chem Phys 114:7483-7496. doi:10.1063/1.1356030

Tolentino TP, Wu J, Zarnitsyna VI, Fang Y, Dustin ML, Zhu C (2008) Measuring diffusion and binding kinetics by contact area FRAP. Biophys J 95:920-930. doi:10.1529/biophysj.107.114447 
Ushiyama S, Laue TM, Moore KL, Erickson HP, McEver RP (1993) Structural and functional-characterization of monomeric soluble P-selectin and comparison with membrane P-selectin. J Biol Chem 268:15229-15237

Veigel C, Bartoo ML, White DC, Sparrow JC, Molloy JE (1998) The stiffness of rabbit skeletal actomyosin cross-bridges determined with an optical tweezers transducer. Biophys J 75:1424-1438. doi:10.1016/S0006-3495(98)74061-5

Veigel C, Molloy JE, Schmitz S, Kendrick-Jones J (2003) Loaddependent kinetics of force production by smooth muscle myosin measured with optical tweezers. Nat Cell Biol 5:980-986. doi: $10.1038 /$ ncb 1060

Williams TE, Nagarajan S, Selvaraj P, Zhu C (2001) Quantifying the impact of membrane microtopology on effective two-dimensional affinity. J Biol Chem 276:13283-13288. doi:10.1074/jbc. M010427200

Wu L, Xiao BT, Jia XL, Zhang Y, Lu SQ, Chen J et al (2007) Impact of carrier stiffness and microtopology on two-dimensional kinetics of $\mathrm{P}$-selectin and P-selectin glycoprotein ligand-1
(PSGL-1) interactions. J Biol Chem 282:9846-9854. doi: 10.1074/jbc.M609219200

Yago T, Leppanen A, Qiu H, Marcus WD, Nollert MU, Zhu C et al (2002) Distinct molecular and cellular contributions to stabilizing selectin-mediated rolling under flow. J Cell Biol 158:787799. doi:10.1083/jcb.200204041

Yago T, Zarnitsyna VI, Klopocki AG, McEver RP, Zhu C (2007) Transport governs flow-enhanced cell tethering through L-selectin at threshold shear. Biophys J 92:330-342. doi:10.1529/ biophysj.106.090969

Zarnitsyna VI, Huang J, Zhang F, Chien YH, Leckband D, Zhu C (2007) Memory in receptor-ligand-mediated cell adhesion. Proc Natl Acad Sci USA 104:18037-18042. doi:10.1073/pnas. 0704811104

Zhang Y, Sun G, Lu S, Li N, Long M (2008) Low spring constant regulates P-selectin-PSGL-1 bond rupture. Biophys J 95:54395448. doi:10.1529/biophysj.108.137141

Zhu C (2000) Kinetics and mechanics of cell adhesion. J Biomech 33:23-33. doi:10.1016/S0021-9290(99)00163-3 